\title{
NATURE OF THE ANODIC TELLURATE WAVE AT THE DROPPING MERCURY ELECTRODE
}

\author{
B. JASELSKIS and J. G. LANESE \\ Department of Chemistry, University of Michigan, Ann Arbor, Michigan
}

(Received 11 September 1961)

\begin{abstract}
Tellurate solutions at the dropping mercury electrode show an adsorption type anodic wave in the vicinity of zero volts vs. S.C.E. The presence of this anodic wave is attributed to depolarization of mercury electrode and the formation of an insoluble mercurous tellurate film given by the reaction $2 \mathrm{Hg}^{\circ}+\mathrm{H}_{6} \mathrm{TeO}_{6} \rightarrow \mathrm{Hg}_{2} \mathrm{H}_{4} \mathrm{TeO}_{6}+2 \mathrm{H}^{+}+2 \mathrm{e}$. Under similar conditions an anodic wave is not observed with tellurite, selenate, selenite and arsenate.
\end{abstract}

AT the dropping mercury electrode tellurate shows a well defined cathodic wave. The nature of this wave and analytical applications have been described by NORTON et al.(1) Closer examination of dilute tellurate solutions reveals the presence of an anodic wave in the vicinity of zero volts vs. S.C.E. in addition to the reported cathodic wave. In a limited concentration range this wave is proportional to the concentration. The nature of this anodic wave is of interest and is described in this paper.

\section{EXPERIMENTAL}

Apparatus. Pularograms were recorded by a Sargent Model XXI polarograph using a thermostated polarographic $\mathrm{H}$ cell with an external reference electrode. Both the saturated calomel and the saturated mercury-mercurous sulphate electrode were used. Oxygen was eliminated by passing a purified nitrogen through the solution.

Materials. Buffer solutions of a desired ionic strength were prepared from the reagent grade chemicals. The ionic strength was adjusted with potassium sulphate. Telluric acid was obtained from the Amend Drug and Chemical Co. and also was prepared from a pure metallic tellurium by the oxidation procedure described in the BRAUER's Handbuch der Praeparativen Anorganischen Chemie. (2) Mercurous tellurate (later referred as a black precipitate) was prepared by electrolysis of tellurate buffered solutions using mercury pool electrode at the potential set slightly more positive than the calomel electrode or by reacting metallic mercury with tellurate without an applied potential.

Polarograms of tellurate solutions. The anodic portion of polarogram was recorded by changing the potential of the dropping mercury electrode from negative towards positive potentials. In this manner smooth curves were obtained. Otherwise at high concentrations of tellurate the anodic curve shows some irregularities.

\section{EXPERIMENTAL RESULTS}

Anodic tellurate waves. In Britton and Robinson buffer solutions at $\mathrm{pH} 10$ in the presence of chloride the tellurate ion shows a well defined anodic wave in the vicinity of zero volts vs. S.C.E. At lower pH's this wave is poorly defined and can be overlooked. Better results can be obtained when the mercury،mercurous sulphate reference electrode is used in the bicarbonate-carbonate buffer solutions containing potassium sulphate. A typical polarogram of a dilute tellurate solution is shown in

(1) E. Norton, R. W. Stoenner and A. I. Medalia, J. Amer. Chem. Soc. 75, 1821 (1953)-

(2) G. Brauer, Handbuch der Praparativen Anorganischen Chemie, pp. 348, 349. Ferdinand Enke, Stuttgart (1954). 
Fig. 1. At higher concentrations of tellurate than $8 \times 10^{-5} \mathrm{M}$ the anodic wave forms a poorly defined prewave. Similar prewave for halide solutions has been observed by KoLTHOFF and OKINAKA ${ }^{(3)}$ and has been attributed to the formation of a monomolecular layer of the mercurous halide.

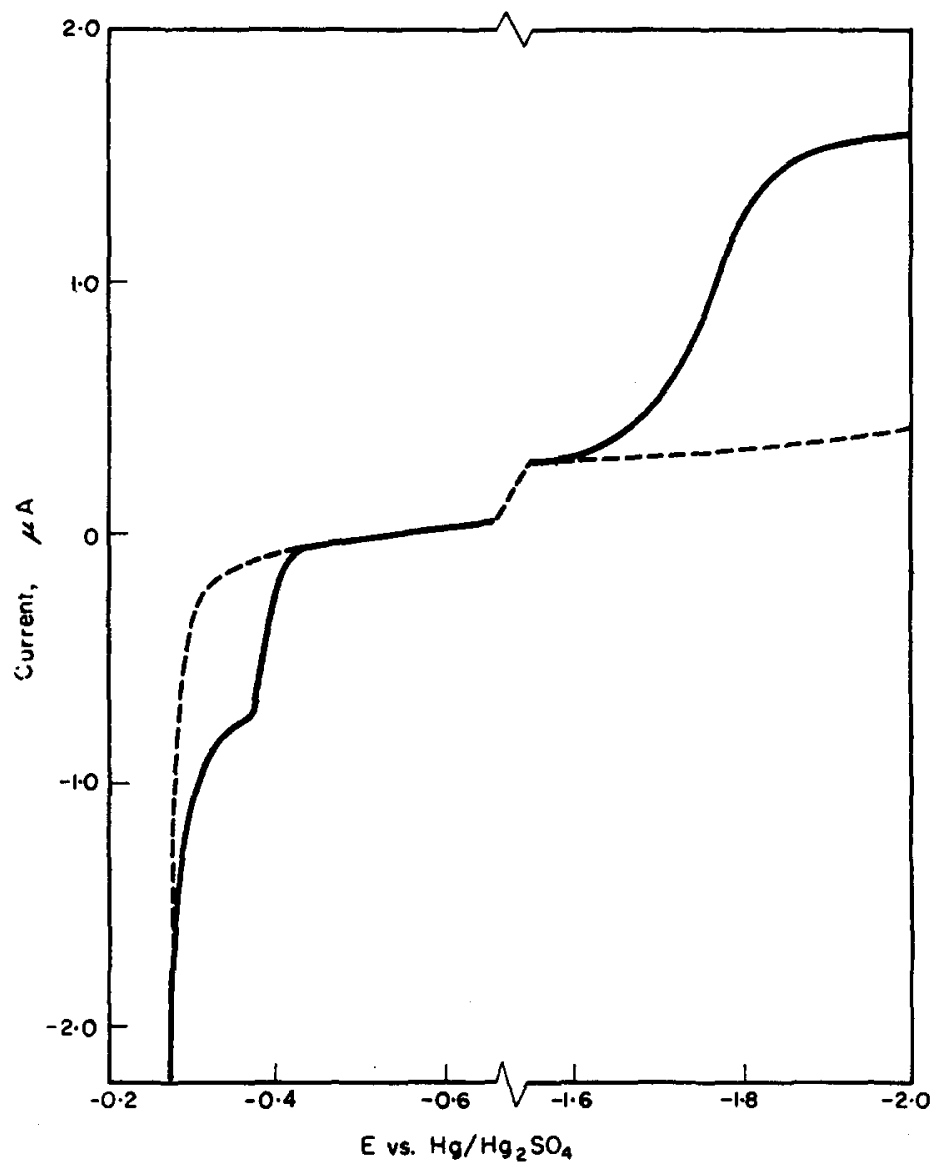

Fig. 1.-Polarogram of $5+10^{-5} \mathrm{M}$ tellurate in bicarbonate-carbonate buffer.

The limiting current is directly proportional to the height of mercury column and it is inversely proportional to the temperature if the concentration of tellurate is above $8 \times 10^{-5} \mathrm{M}$. At temperatures over $65^{\circ} \mathrm{C}$ the anodic wave disappears. Temperature effect on the anodic diffusion current changes somewhat with the concentration of tellurate. Changes become pronounced when the tellurate ion concentration is less than $8 \times 10^{-5} \mathrm{M}$.

Electrocapillary characteristics. Electrocapillary characteristics of the tellurate solutions are considerably different from those of the supporting electrolyte alone as shown in Fig. 2. The electrocapillary curve for the tellurate solution shows a maximum at approximately the half-wave potential of the anodic wave and also a broad shoulder in the vicinity of the electrocapillary maximum of the supporting electrolyte. Similar electrocapillary behaviour has been reported by BENESCH and

(3) I. M. Kolthoff and Y. Okinaka, J. Amer. Chem. Soc. 83, 47 (1961). 
BENESCH ${ }^{(4)}$ for the reduction of organic mercury compounds. The drop time of mercury decreases progressively by increasing the concentration of tellurate until a saturation concentration is reached, approximately $8 \times 10^{-5} \mathrm{M}$. The addition of various maximum suppressors have no effect on the diffusion current. Some effect is observed with Triton $X-100$ and camphor. In the presence of 0.1 per cent of Triton X-100 electrocapillary curve for tellurate is somewhat depressed but the general shape remains the same as for the tellurate alone. The results indicate that mercurous tellurate and Triton X-100 are both absorbed simultaneously and that no replacement of the one film by the other takes place. In the presence of chelating agents such as ethylenediaminetetracetate the anodic wave disappears.

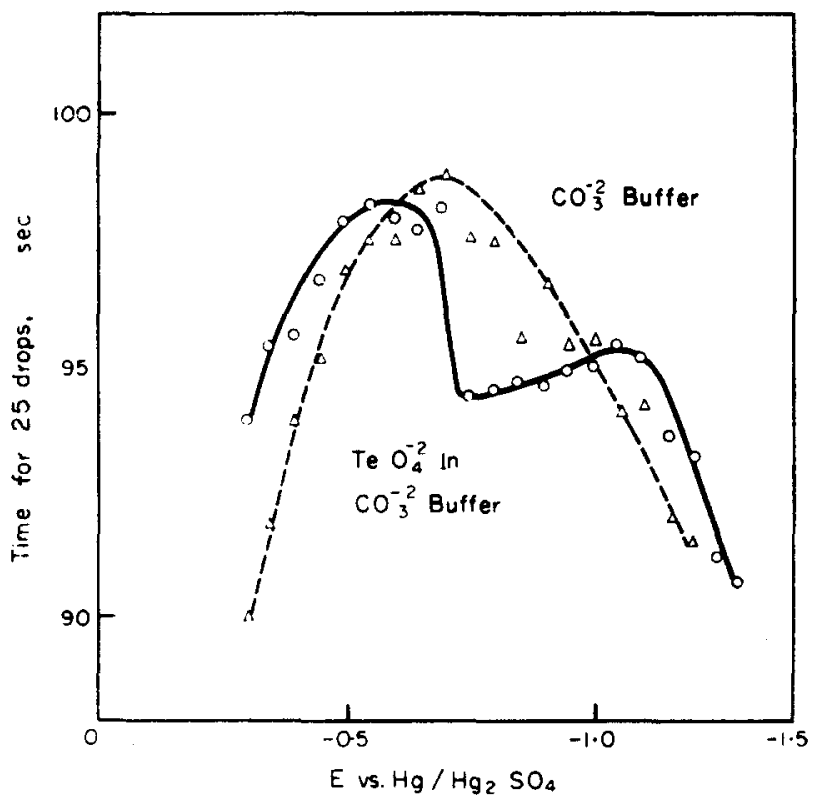

FIG. 2.-Electrocapillary curves: $0 \cdot 1 \mathrm{M}$ carbonate-bicarbonate and $0 \cdot 1 \mathrm{M}$ potassium sulphate buffer solution in the presence and in the absence of tellurate.

Dependence of the limiting current and half-wave potential on concentration, $p H$ and ionic strength. The limiting current for the anodic wave increases with the concentration of tellurate until a saturation value is reached. Approximately the same values of saturation currents are obtained with the buffer solutions investigated. However, the dependence of a limiting current on concentration is somewhat affected by the composition of the buffer as shown in Fig. 3. In phosphate buffer solutions the reproducibility of the limiting current is poor. The half-wave potential for the anodic wave becomes more negative with the increasing $\mathrm{pH}$. It changes in a linear manner with $\mathrm{pH}$. The slope for the curve of the half-wave potential vs. $\mathrm{pH}$ is approximately 0.062 . The half-wave potential also varies in a linear manner with the logarithm of the tellurate ion concentration. The slope for this curve is approximately 0.020 . Variation of the half-wave potential with the $\mathrm{pH}$ and with the logarithm of

(4) R. E. Benesch and R. Benesch. J. Phys. Chem. 56, 648 (1952). 


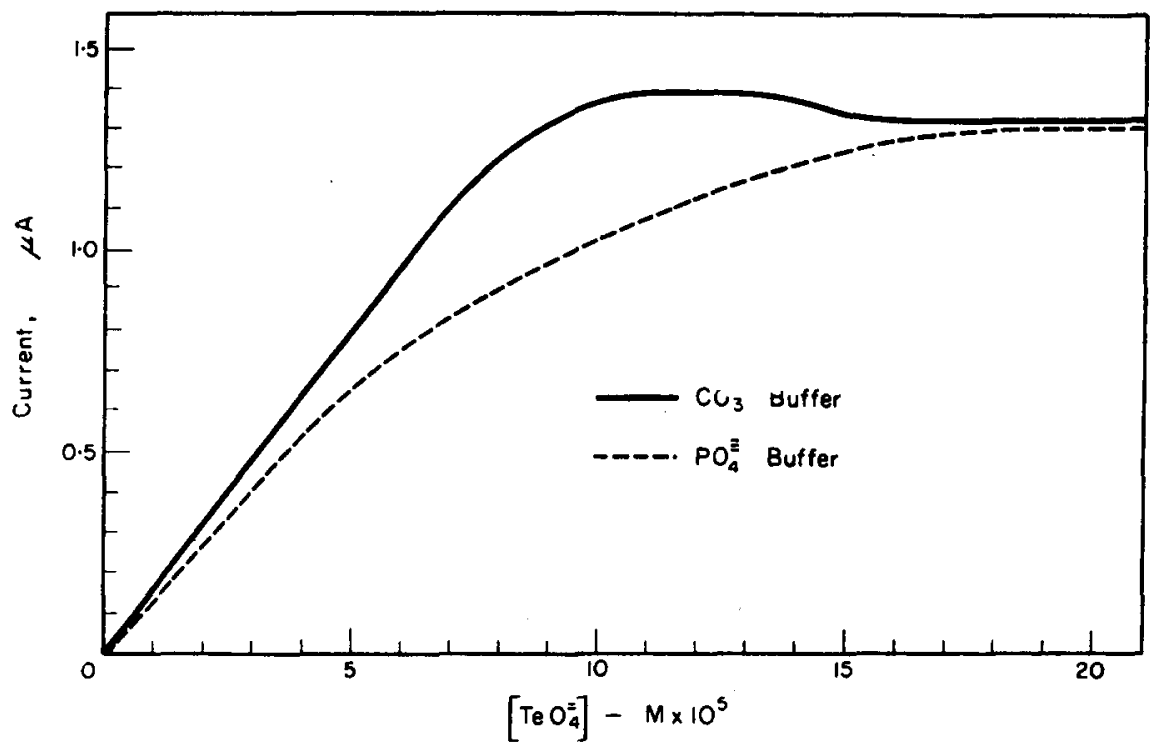

FIG. 3.-Height of the anodic wave as a function of tellurate ion concentration.

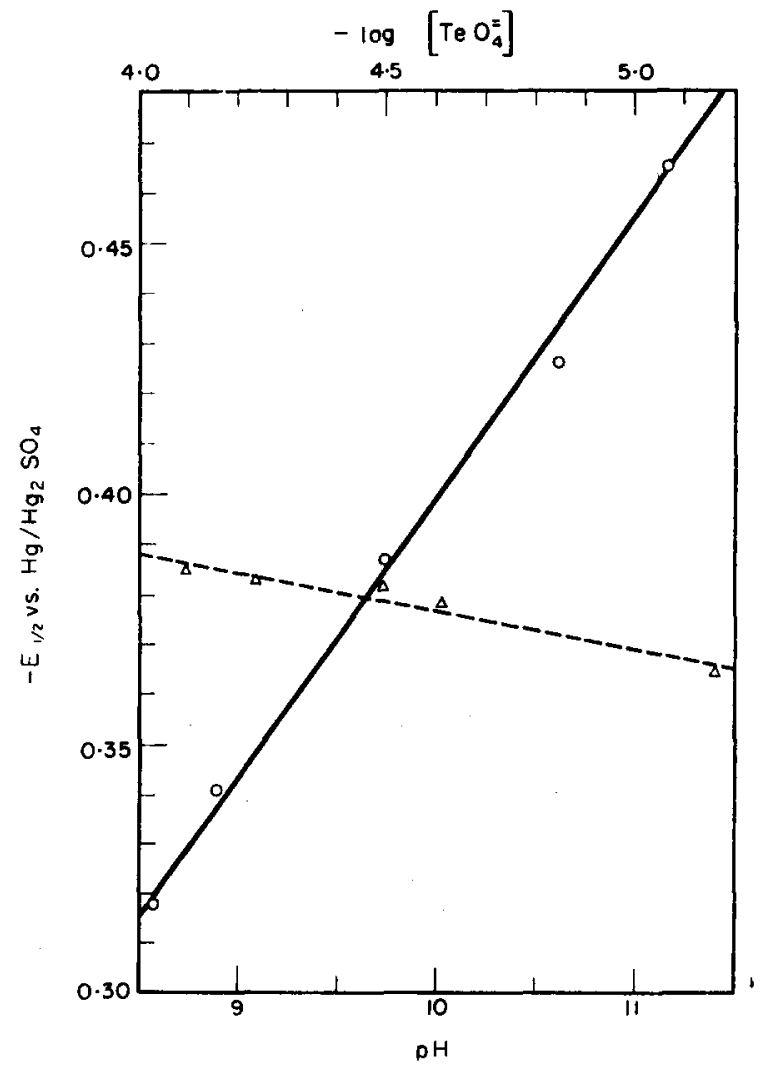

FIG. 4.-Half-wave potential of the anodic wave as a function of $\mathrm{pH}$ and the negative logarithm of the tellurate ion concentration. (Solid line- $E_{\frac{1}{3}}$ vs. $\mathrm{pH}$ and the dashed line$E_{\frac{1}{2}}$ vs. $-\log \left(\mathrm{TeO}_{4}^{-}\right)$. 
the tellurate ion concentration is shown in Fig. 4. The limiting current and the half-wave potential dependence on the concentration of tellurate and $\mathrm{pH}$ is shown in Table 1.

Table 1.-Polarographic measurements on tellurate solutions

\begin{tabular}{cccc}
\hline $\begin{array}{c}\text { Concentration } \\
\text { of } \\
\text { tellurate } \\
(\mathrm{mM})\end{array}$ & $\begin{array}{c}\text { Anodic } \\
\text { diffusion current } \\
i(\mu \mathrm{A})\end{array}$ & $\begin{array}{c}\text { Anodic half-wave } \\
\text { potential* } \\
(\mathrm{v})\end{array}$ & $\begin{array}{c}\mathrm{pH} \\
\text { of the supporting } \\
\text { electrolyte }\end{array}$ \\
\hline 0.0069 & 0.10 & 0.364 & $9.70 \dagger$ \\
0.0245 & 0.37 & 0.378 & $"$, \\
0.0323 & 0.46 & 0.381 & $"$, \\
0.0578 & 0.88 & 0.383 & $8.58 \dagger$ \\
0.0804 & 1.19 & 0.385 & $8.89 \dagger$ \\
0.050 & 0.71 & 0.318 & $9.74 \dagger$ \\
0.050 & 0.69 & 0.341 & $10.61 \dagger$ \\
0.050 & 0.71 & 0.387 & $11.16 \dagger$ \\
0.050 & 0.71 & 0.426 & \\
0.050 & 0.73 & 0.465 & \\
\hline
\end{tabular}

* Anodic potentials are measured against mercury-mercurous sulphate reference electrode.

$\dagger$ All buffer solutions contain potassium bicarbonate-carbonate and potassium sulphate.

Variation of the ionic strength by a factor of ten has no effect on the half-wave potential.

\section{DISCUSSION}

Diffusion current dependence on concentration, electrocapillary characteristics and visual observation of mercury droplets suggest that the anodic wave is due to the formation of an isoluble film and adsorption of tellurate ions. During polarographic scanning of tellurate solutions mercury droplets are covered with a black precipitate. LINGANE and NIEDRACH ${ }^{(5)}$ report a similar precipitate for the reduction of the tellurite ion in the vicinity of the tellurite reduction wave. The nature of this precipitate has been attributed to the formation of the metallic tellurium. However, the black precipitate, which is formed by the reaction of tellurate with the metallic mercury without or with an electrical potential applied, is quite different from that reported by LINGANE and NIEDRACH. ${ }^{(5)}$ This precipitate contains mercurous and tellurate ions. The composition of this precipitate corresponds to 65 per cent mercury and 18 per cent tellurium. The empirical formula of the black precipitate cannot be ascertained unambiguously since it appears to vary somewhat with the conditions of the preparation. These resuts compare favourably with the mercurous tellurate. $\left(\mathrm{Hg}_{2} \mathrm{H}_{4} \mathrm{TeO}_{6} ; 64 \% \mathrm{Hg}: 20 \cdot 3 \% \mathrm{Te}\right.$.)

Formation of anodic waves at the dropping mercury electrode by halide solutions have been described by Kolthopf and MilleR (6) and by Ravenda. ${ }^{(7)}$ Similar

(5) J. J. Lingane and L. M. Niedrach, J. Amer. Chem. Soc. 71, 196 (1949).

(6) I. M. Kolthoff and C. S. Miller, J. Amer. Chem. Soc. 63, 1405 (1941).

(7) J. Ravenda, Coll. Trav. Chim. Tchecosl. 6, 453 (1934). 
anodic waves have been reported for periodate by JENSOVSKY ${ }^{(8)}$ and for organic mercury compounds by BENESCH and BENESCH. ${ }^{(4)}$ In all cases the mercury electrode is depolarized by the formation of an insoluble mercurous salt. Thus, the nature of the anodic wave of tellurate solutions at the dropping mercury electrode is attributed to the depolarization of mercury and the formation of an insoluble film of mercurous tellurate by the reaction:

$$
2 \mathrm{Hg}^{\circ}+\mathrm{H}_{6} \mathrm{TeO}_{6} \rightarrow \mathrm{Hg}_{2} \mathrm{H}_{4} \mathrm{TeO}_{6}+2 \mathrm{H}^{+}+2 e .
$$

Hence the half wave potential is related to the concentration of tellurate and $\mathrm{pH}$ by the equation:

$$
E_{\frac{1}{2}}=\text { constant }-\frac{0.0591}{2} \log \frac{\mathrm{Te}(\mathrm{VI})}{2}-0.0591 \mathrm{pH} .
$$

Thus the half wave potential should vary as the logarithm of the tellurate concentration and as the $\mathrm{pH}$. Experimentally determined slopes for these variations are $\mathbf{0 . 0 2 0}$ and 0.062 respectively.

The reversibility of the anodic depolarization by tellurate has been evaluated from the slope in the plot of the logarithm of $\left(i_{d}-i\right)$ vs. potential of the dropping mercury electrode:

$$
E_{\text {d.m.e. }}=\text { constant }-\frac{0.0591}{2} \log \left(i_{d}-i\right)
$$

The slope for the anodic iellurate wave in the concentration ranges below $8 \times 10^{-5}$ is approximately $60 \mathrm{mV}$. For more concentrated tellurate solutions than $8 \times 10^{-5} \mathrm{M}$ two slopes are obtained; $60 \mathrm{mV}$ for the major portion of the wave and $125 \mathrm{mV}$ for the prewave. These results clearly indicate that the tellurate anodic wave is irreversible, and the adsorption wave is still much more drawn out.

All these observations suggest that the anodic tellurate wave is a surface-film limited wave not, strictly speaking, an adsorption wave.

(8) L. Jensovsky, Coll. Trav. Chim. Tchecosl. 22, 311 (1957). 